Aim of the study: To evaluate the feasibility of whole-brain radiotherapy (WBRT) with a simultaneous integrated boost (SIB) by forward intensitymodulated radiation therapy (IMRT) in patients with 1-3 brain metastases. Material and methods: Two forward IMRT plans were implemented among 18 patients. In plan $\mathrm{A}$, the prescribed dose was 30 Gy to the whole brain $\left(\right.$ PTV $\left._{\text {WBRT }}\right)$ and 50 Gy to individual brain metastases (PTV ${ }_{\text {boost }}$ ) delivered simultaneously in 10 fractions. In plan B, the prescribed dose was 30 Gy to the PTV ${ }_{\text {WBRT }}$ and 40 Gy to the PTV boost. $_{\text {Plans }}$ were evaluated with regard to conformation number $(\mathrm{CN})$, prescription isodose volume to target volume ratio (PITV), target coverage (TC), homogeneity index ( $\mathrm{HI})$, and the volume receiving at least $95 \%$ of the prescribed dose $\left(V_{95}\right)$. Plan $A$ was implemented for 5 of these patients, and plan B was used for the remaining patients.

Results: The mean values of CN, PITV, $\mathrm{TC}$, and $\mathrm{HI}$ for the PTV ${ }_{\text {boost }}$ were 0.71 , $1.32,0.97$, and 0.07 , respectively, for plan $A$ and $0.65,1.47,0.97$, and 0.05 , respectively, for plan $B$. The mean values of TC, $\mathrm{HI}$, and $\mathrm{V}_{95}$ for the PTV were $0.98,0.45$, and $99.71 \%$, respectively, for plan A and $0.97,0.27$, and $99.61 \%$, respectively, for plan B. All patients completed the planned radiotherapy (RT) schedule with no acute and late RT-related toxicity greater than grade 2 .

Conclusions: It is feasible to deliver WBRT with a SIB via forward IMRT for patients with 1-3 brain metastases with good dose conformity and acceptable toxicity.

Key words: simultaneous integrated boost, forward intensity-modulated radiation therapy, brain metastases.

Contemp Oncol (Pozn) 2014; 18 (3): 187-191 DOI: $10.5114 /$ wo. 2014.43156

\section{Feasibility of simultaneous integrated boost with forward intensity- modulated radiation therapy for multiple brain metastases}

\author{
Lingqin $\mathrm{Ni}^{1}$, Xiaodong Liang ${ }^{2}$
}

${ }^{1}$ Taizhou Central Hospital, Taizhou, PR China

${ }^{2}$ Radiation Oncology Department, Hangzhou Cancer Hospital, Hangzhou, PR China

\section{Introduction}

Brain metastases are a common problem in adults with cancers, and the results are generally unsatisfactory despite the rapid development of multimodality treatments $[1,2]$. Non-randomised studies suggest that whole-brain radiotherapy (WBRT) increases the median survival time by 3-4 months compared to approximately 1 month without treatment and 2 months with corticosteroids alone [3]. The Radiation Therapy Oncology Group (RTOG) 9508 trial randomised 333 patients with 1-3 cerebral metastases to standard WBRT either with or without a stereotactic radiation (SRS) boost and demonstrated a survival benefit for patients with a single brain metastasis and an improved quality of life for all patients who received the SRS boost [4]. Other reports suggested that combined WBRT and SRS for multiple metastases significantly improves the control of brain disease [5, 6]. It is beneficial to deliver a higher radiation dose to cerebral metastases in addition to standard WBRT in selected patients, based on the aforementioned evidence.

However, SRS is not available in most radiation departments, and the procedure is time consuming and expensive. Several authors have investigated the feasibility of WBRT with a simultaneous integrated boost (SIB) via intensity-modulated radiation therapy (IMRT) in patients with brain metastases. Bauman et al. [7] confirmed the feasibility of an SIB to individual brain metastases during a course of WBRT using helical tomotherapy (HT) IMRT. Lagerwaard et al. [8] describe the delivery of WBRT and SIB to multiple brain metastases via RapidArc as a rapid and accurate technique. In addition, RapidArc achieved a higher conformity index than the conventional summation of WBRT and SRS. Edwards et al. [9] treated 11 patients with brain metastases by IMRT to deliver an SIB during a standard $10 \times 3$ Gy fractionated WBRT course, and they found no adverse effects and good early effects with regard to local control. With IMRT technology, delivering dose boosts to metastases could provide the advantages of SRS during WBRT and obviate the need for the additional procedure. The SIB technique also keeps overall treatment courses short to minimise tumour cell repopulation and patient inconvenience. However, helical tomotherapy and RapidArc are not available at many radiation centres, unlike forward IMRT.

We previously reported the preliminary dosimetric results of an SIB during WBRT delivered via forward IMRT [10]. In this study, we implemented 2 plans in 18 patients with 1-3 brain metastases and compared the plans with respect to conformity and treatment delivery time. We also treated these patients with planned protocols and evaluated the acute and late treatment-related toxicities prospectively. 


\section{Material and methods}

This study was approved by our Research Ethics Board. Eighteen patients with 1-3 brain metastases were included between March 2010 and December 2010. The patient eligibility criteria for this study were as follows: histologically proven cancer and imaging confirmation of 1-3 brain metastases on pretreatment contrast-enhanced computed tomography (CT) or magnetic resonance imaging. The exclusion criteria for this study were as follows: > 3 lesions, metastases close (within $5 \mathrm{~mm}$ ) to the brainstem or optic apparatus, cytological or imaging evidence of leptomeningeal metastases with other histologic confirmation of malignancy, lack of informed consent, previous cranial radiotherapy (RT) and any contraindications for CT contrast. The patient characteristics are shown in Table 1.

All of the patients were positioned supine in a custom-made mask. Planning CT scans (GE Bright Speed Elite 16 slice CT scanner) with intravenous contrast were obtained with a 2.5-mm slice thickness. The plans were applicable to forward IMRT. The number of metastases ranged from 1 to 3 with a mean of 1.72 .

Table 1. Patient characteristics

\begin{tabular}{|c|c|}
\hline Variable & \\
\hline $\begin{array}{l}\text { Age (years) } \\
\text { median } \\
\text { range }\end{array}$ & $\begin{array}{c}60 \\
41-78\end{array}$ \\
\hline $\begin{array}{l}\text { Gender } \\
\text { male } \\
\text { female }\end{array}$ & $\begin{array}{c}11 \\
7\end{array}$ \\
\hline $\begin{array}{c}\text { RPA } \\
1 \\
2 \\
3\end{array}$ & $\begin{array}{c}0 \\
6 \\
12\end{array}$ \\
\hline $\begin{array}{l}\text { KPS } \\
\geq 70 \\
<70\end{array}$ & $\begin{array}{c}6 \\
12\end{array}$ \\
\hline $\begin{array}{l}\text { GPA } \\
\text { median } \\
\text { range }\end{array}$ & $\begin{array}{c}1 \\
0.5-2\end{array}$ \\
\hline $\begin{array}{l}\text { Primary } \\
\text { lung cancer } \\
\text { rectal cancer } \\
\text { breast cancer }\end{array}$ & $\begin{array}{c}13 \\
3 \\
2\end{array}$ \\
\hline $\begin{array}{l}\text { Primary tumour status } \\
\text { controlled } \\
\text { uncontrolled }\end{array}$ & $\begin{array}{c}4 \\
14\end{array}$ \\
\hline $\begin{array}{l}\text { Number of metastases } \\
1 \\
2 \\
3\end{array}$ & $\begin{array}{l}8 \\
5 \\
5\end{array}$ \\
\hline $\begin{array}{l}\text { PTV of brain metastases } \\
\quad<20 \mathrm{cc} \\
\geq 20 \mathrm{cc}\end{array}$ & $\begin{array}{l}9 \\
9\end{array}$ \\
\hline $\begin{array}{l}\text { Chemotherapy and/or TKI post WBRT } \\
\text { yes } \\
\text { no }\end{array}$ & $\begin{array}{c}5 \\
13\end{array}$ \\
\hline
\end{tabular}

RPA - recursive partitioning analysis; GPA - graded prognostic assessment; PTV - planning target volume; TKI - tyrosine kinase inhibitor; WBRT - wholebrain radiotherapy

\section{Target definition and treatment planning}

The PTV ${ }_{\text {boost }}$ was created by adding a $3-\mathrm{mm}$ margin to the visible metastases $\left(\mathrm{GTV}_{\text {boost }}\right)$. The PTV $\mathrm{V}_{\text {WBRT }}$ was derived from the whole brain plus the addition of a 3-mm symmetric margin. Two types of forward plans were generated for each patient on the Pinnacle 6.2 Treatment Planning System (TPS). In plan A, the prescribed dose was 30 Gy to a $95 \%$ volume of the PTV WBRT $_{\text {and }} 50$ Gy to a $95 \%$ volume of the PTV ${ }_{\text {boost }}$, delivered simultaneously in 10 fractions. In plan B, the prescribed dose was 30 Gy to a $95 \%$ volume of the PTV WBRT $_{\text {Th }}$ and 40 Gy to a $95 \%$ volume of the PTV boost

For all patients, treatment plans were generated with 6-MV photons by using multi-leaf collimation with a leaf width of $10 \mathrm{~mm}$ (Siemens Primus H). The PTV ${ }_{\text {WBRT }}$ was irradiated by parallel, opposed conformal photon beams, and the PTV ${ }_{\text {boost }}$ was boosted with several oblique, coplanar, conformal photon beams sparing the lens and brainstems. Beam weight and directions were manually optimised. The dose rate for treatment delivery was 200 monitor units (MUs) per minute.

\section{Plan evaluation criteria}

Treatment plans were evaluated regarding the following criteria: conformation number (CN), prescription isodose to target volume ratio (PITV), target coverage (TC), homogeneity index $(\mathrm{HI})$, the volume that received at least 95\% of the prescribed dose $\left(\mathrm{V}_{95}\right)$ and treatment delivery time. The PTV boost $_{\text {and PTV }}$ WBRT were used as the target volume.

Dose conformity was characterised by the $\mathrm{CN}$ as proposed by van't Riet et al. [11]. The CN is defined as follows: $\mathrm{CN}=\left(\mathrm{V}_{\mathrm{T} \text {,pres }} \times \mathrm{V}_{\mathrm{T} \text {, pres }}\right) /\left(\mathrm{V}_{\mathrm{T}} \times \mathrm{V}_{\text {pres }}\right)$, where $\mathrm{V}_{\mathrm{T} \text {, pres }}$ is the volume within the target receiving a dose of no less than the prescription dose, $V_{T}$ is the PTV and $V_{\text {pres }}$ is the volume receiving a dose of no less than the prescription dose. The CN ranged from 0 to 1 . A higher $C N$ value indicates better conformity.

The PITV is defined as follows [12]: PITV $=V_{\text {pres }} / V_{T}$. A PITV $<1.0$ indicates that the target volume is not completely covered by the prescription line, whereas a value $>1.0$ indicates that the prescription line covers too much.

The $T C$ is defined as follows: $T C=V_{T, p r e s} N_{T}$. A TC of 1.0 indicates perfect coverage.

The $\mathrm{HI}$ is defined as follows: $\mathrm{HI}=\left(\mathrm{D}_{2 \%}-\mathrm{D}_{98 \%}\right) / \mathrm{D}_{\text {median }}[13]$, where $D_{2 \%}$ is the dose delivered to $2 \%$ of the target volume, $D_{98 \%}$ is the dose delivered to $98 \%$ of the target volume and $\mathrm{D}_{\text {median }}^{98 \%}$ is the median dose of the target volume. A lower $\mathrm{HI}$ indicates a more homogenous dose distribution in the target volume.

The $V_{95}$ is defined as the volume receiving at least $95 \%$ of the prescribed dose in the PTV. The treatment delivery time was calculated while the dose rate for treatment delivery was 200 MUs per minute.

\section{QA of forward IMRT plans and RT schedule}

All plans were delivered on a Siemens Primus $\mathrm{H}$ linear accelerator and measured in a plastic water phantom by using the MatriXX 2D ion chamber array detector (IBA, Schwarzenbruck, Germany). The dose distribution measured by the MatriXX device was compared with that cal- 
culated by TPS on the same plane using gamma criteria of a $3 \%$ dose and a $3-\mathrm{mm}$ distance to agreement. Five of the patients in RPA class 2 with a summated PTV boost $_{\text {of }}$ less than $20 \mathrm{~cm}^{3}$ were selected to be treated with plan A, and the other 13 patients were treated with plan B. Acute toxicity (within 3 months after the commencement of RT) and late toxicity (beyond 3 months after the commencement of RT) were scored according to the RTOG scoring system [14, 15]. Toxicity was assessed during RT, 1 month after the end of RT and every 2 months after that. Anticonvulsants, mannitol and steroids were used as and when needed.

\section{Statistical analysis}

A comparison of the indices between plans A and B was performed by an independent-samples t-test, using SPSS17 software (SPSS Chicago, IL) at a 95\% confidence level.

\section{Results}

Table 2 shows the values of indices for the PTV ${ }_{\text {boost? }}$ and Table 3 shows those for the PTV ${ }_{\text {WBRT }}$. MU and expected treatment delivery time data are shown in Table 4. The median values of $\mathrm{CN}$ were 0.74 and 0.66 for plans $A$ and $\mathrm{B}$, respectively. The entire volume of the PTV ${ }_{\text {boost }}$ received at least $95 \%$ of the prescribed dose in all cases. The maximum point doses were less than $110 \%$ of the pre-

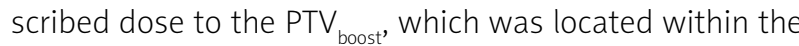
PTV ${ }_{\text {boost }}$ in all cases. Ten beams were used on average in these plans. All patients completed the planned RT schedule without treatment interruption. Radiation-induced toxicity was minimal (Table 5) during the follow-up period (median, 7.8 months; range, 1.8-25 months). No patients presented with early or late events $>$ grade 2 . At the end of the last follow-up, 2 of the 18 patients were alive. The median survival time was 7 months (range, 1.8-25 months).

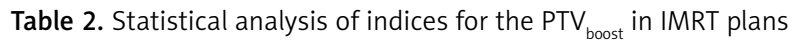

$\begin{array}{lcccc}\text { Indices } & \text { Plan A } & \text { Plan B } & t \text { value } & p \text { value } \\ \text { CN } & 0.71 \pm 0.09 & 0.65 \pm 0.09 & 2.096 & 0.044 \\ \text { PITV } & 1.32 \pm 0.20 & 1.47 \pm 0.24 & 2.109 & 0.042 \\ \text { TC } & 0.97 \pm 0.02 & 0.97 \pm 0.02 & 1.834 & 0.075 \\ \text { HI } & 0.07 \pm 0.04 & 0.05 \pm 0.03 & 0.1 & 0.921\end{array}$

CN - conformation number; PITV - prescription isodose to target volume ratio; TC-target coverage; $\mathrm{HI}$-homogeneity index

The two-tailed $p$ values were results from independent-sample t-tests.

Table 4. Treatment delivery time

\begin{tabular}{|lcccc|}
\hline Indices & Plan A & Plan B & $t$ value & $p$ value \\
MU & 747.72 & 538.44 & 4.455 & 0.000 \\
& \pm 179.41 & \pm 86.76 & & \\
& & & & \\
TDT (min) & $3.74 \pm 0.90$ & $2.70 \pm 0.43$ & 4.456 & 0.000
\end{tabular}

MU - monitor units; TDT - treatment delivery time

The two-tailed $p$ values were results from independent-samples $t$-tests.

\section{Discussion}

In this article, the prescribed doses to the PTV ${ }_{\text {WBRT }}$ were similar for the 2 plans, whereas the prescribed doses to

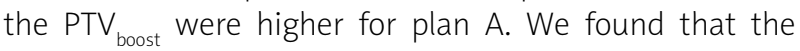
mean values of CN and PITV for the PTV ${ }_{\text {boost }}$ were higher for plan A. Conformity was influenced by the prescribed doses, and it appears that conformity was better in plan A with regard to CN and PITV. The dose schedule should be considered when interpreting the conformity of different radiotherapy plans.

To assess the quality of our plans, we compared our results with previous studies on SRS and inverse IMRT. For metastases, a measure of the quality of the dose distribution can be assessed by the CN proposed by van't Riet et al. [11]. The median and mean values of CN were 0.74 and 0.66 , respectively, for plan A and 0.71 and 0.65 , respectively, for plan B in this study. Nakamura et al. [16] compiled dose conformity statistics for patients treated with gamma knife radiosurgery and found a median $\mathrm{CN}$ value of 0.56 for all lesions. Gutiérrez et al. [13] determined that composite tomotherapy plans achieved both homogeneous whole-brain dose distributions equivalent to those of conventional WBRT and radiosurgically equivalent dose distributions to individual metastases. The mean CN value was 0.59 in that report. Peñagarícano et al. [17] reported that the values of CN vary from 0.547 to 0.644 and from 0.507 to 0.696 in patients with single brain metastases planned by helical tomotherapy and SRS, respectively. Dose conformity in this paper was superior to that in previous studies, including those of SRS and helical tomotherapy. Nakamura et al. [16] noted worse conformity for smaller target volumes. Similarly, Gutiérrez et al. [13] found that the mean CN values were $60 \%$ higher (0.444 vs. 0.708) for volumes greater than $2.05 \mathrm{~cm}^{3}$. However, all of the patients PTV boost $_{\text {values were }}$ greater than $2.05 \mathrm{~cm}^{3}$ in this paper, which may explain

Table 3. Statistical analysis of indices for PTV $\mathrm{WBRT}_{\text {in IMRT plans }}$

\begin{tabular}{lcccc} 
Indices & Plan A & Plan B & $t$ value & $p$ value \\
PITV & $1.18 \pm 0.08$ & $1.15 \pm 0.06$ & 1.269 & 0.213 \\
HI & $0.45 \pm 0.11$ & $0.27 \pm 0.06$ & 6.047 & 0.000 \\
TC & $0.98 \pm 0.02$ & $0.96 \pm 0.02$ & 2.33 & 0.026 \\
$\mathrm{~V}_{95}(\%)$ & $99.71 \pm 0.24$ & $99.61 \pm 0.30$ & 1.668 & 0.114 \\
\hline
\end{tabular}

PITV - prescription isodose to target volume ratio; $\mathrm{HI}$ - homogeneity index; TC - target coverage; $V_{95}$ - the volume receiving at least $95 \%$ of the prescribed dose in the PTV

The two-tailed $p$ values were results from independent-sample t-tests.

Table 5. Radiotherapy related toxicities

\begin{tabular}{|ccccc|}
\hline Toxicities & Grade $\mathbf{1}$ & Grade 2 & Grade 3 & Grade 4 \\
\hline Acute toxicities & & & & \\
leukopenia & 2 & 1 & - & - \\
alopecia & 2 & 7 & - & - \\
dermatitis & 4 & - & - & - \\
anorexia & 2 & - & - & - \\
Late toxicities & & & & \\
alopecia & 3 & - & - & - \\
\hline
\end{tabular}


our better dose conformity regarding $\mathrm{CN}$. In addition, the mean $\mathrm{CN}$ values in this study were close to those achieved in patients with PTV ${ }_{\text {boost }}$ values exceeding $2.05 \mathrm{~cm}^{3}$ as reported by Gutiérrez et al. [13]. Tomita et al. [18] treated 24 patients with 1-4 brain metastases with IMRT using helical tomotherapy, and the mean values of $\mathrm{CN}$ and $\mathrm{HI}$ were 0.75 and 0.063 , respectively. The dose schedule of plan $A$ in this study and the values of $\mathrm{CN}$ and $\mathrm{HI}$ were similar to the findings reported by Tomita et al. In addition, the results are in line with those of our previous technique report [10].

PITV and TC are other common evaluation criteria to assess the quality of the dose distribution. The mean PITV values were 1.32 and 1.47 for plans $A$ and $B$, respectively. Gutiérrez et al. [13] found that their technique is capable of producing similar or better conformity than gamma knife radiosurgery for target volumes exceeding $1.5 \mathrm{~cm}^{3}$. Gutiérrez et al. also reported mean PITV values of 2.12 and 1.21 for $P T V_{\text {boost }}<2.05 \mathrm{~cm}^{3}$ and $P T V_{\text {boost }} \geq 2.05 \mathrm{~cm}^{3}$, respectively [13]. Lagerwaard [8] reported that integrated plans of WBRT and boosts to multiple brain metastases by volumetric modulated arc therapy (VMRT) had a higher conformity index than the conventional summation of WBRT and SRS boost, as the mean values of $\mathrm{Cl}$ for the 2 strategies were 1.3 and 2.1, respectively. The formulas of $\mathrm{Cl}$ and PITV were identical. Thus, our technique is comparable to helical tomotherapy and VMRT regarding PITV values. TC val-

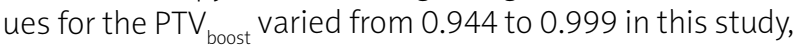
which is comparable to those planed by SRS [16] and helical tomotherapy [13]. It should be noted that $\mathrm{CN}$ is a better quality measure than the PITV ratio and TC value because it accounts for both the PTV and tissue outside the PTV. In addition, the entire volume of the PTV boost received at least $95 \%$ of the prescribed dose in all cases in this study. What is more, the maximum doses were less than $110 \%$ of the prescribed dose to the PTV boost $_{\text {, }}$ whereas all of the hot spots were within the PTV $\mathrm{boost}_{\text {. }}$

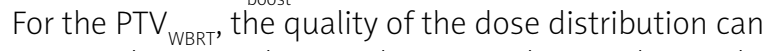
be assessed using indices such as $\mathrm{HI}$ and TC. In this study, the mean values of $\mathrm{HI}$ for the PTV WBRT $_{\text {were }} 0.45$ and 0.27 for plans A and B, respectively, which were not worse than those reported by Gutiérrez et al. [13]. The mean TC values were 0.98 and 0.97 for plans $A$ and $B$, respectively, in this study; findings that were also comparable to the mean TC value of 0.959 reported by Gutiérrez et al. [13]. In addition, the mean values of $V_{95}$ were 0.9971 and 0.9961 for plans

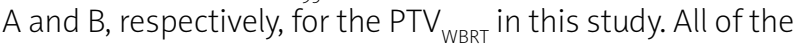
criteria are also satisfactory for the whole brain.

The treatment time for brain metastases depends on the prescribed dose and the technique used. Peñagarícano et al. [17] reported that the treatment time for helical tomotherapy ranged from 30 to 49 minutes when the prescribed doses ranged from 16 to 20 Gy in a single fraction. Bauman et al. [7] reported that the treatment time ranged from 9 to 11 minutes for a single fraction, whereas they prescribed a total intralesional dose of 60 Gy with a surrounding whole-brain dose of 30 Gy in 10 fractions. The mean estimated treatment times were 3.74 and 2.70 minutes for plans $A$ and $B$, respectively, for a single fraction in this study. Therefore, our technique is comparable with helical tomotherapy concerning treatment time.
The median OS was 7 months with no serious toxicities in the present study. The results are promising, and further study is warranted. Although SIB is an emerging technique, the best dose schedule has not been defined, and different schedules were applied in previous studies. The delivery of 60 Gy in 10 fractions to 1-3 brain metastases synchronously during 30-Gy WBRT was found to be feasible and safe in a phase I trial by Rodrigues et al. [19]. The median OS was 5.29 months and no grade $3-5$ toxicities were reported by Rodrigues et al.; findings in line with the results in the present study. Edwards et al. [9] treated patients with bulky brain metastases with an SIB of 40 Gy during 30-Gy WBRT, and the preliminary results are promising with no acute or subacute complications. Tomita et al. [18] delivered 50 Gy to brain metastases and 30 Gy to the whole brain simultaneously in patients with 1-4 brain metastases. No serious complications were documented in any of the studies, indicating that WBRT with an SIB is safe for the aforementioned schedules.

In conclusion, forward IMRT plans of WBRT and an SIB to multiple brain metastases result in highly conformal dose distributions. It appears that our technique is capable of producing comparable conformity as helical tomotherapy regarding CN, PITV, TC and $\mathrm{HI}$. In addition, all patients tolerated the treatment schedule well with no serious toxicity. Therefore, this technique could be useful at radiation centres at which more expensive modalities are not available. A prospective, more advanced trial with more rigorous reporting and data monitoring is required.

The authors declare no conflict of interest.

\section{References}

1. Eichler AF, Loeffler JS. Multidisciplinary management of brain metastases. Oncologist 2007; 12: 884-98.

2. Rolski J, Karczmarek-Borowska B, Smietana A. The possibility of lapatinib treatment for breast cancer patients with central nervous system metastases. Case study and literature review. Contemp Oncol (Pozn) 2012; 16: 582-5.

3. Tsao MN, Lloyd N, Wong RK, Chow E, Rakovitch E, Laperriere N, $\mathrm{Xu} \mathrm{W}$, Sahgal A. Whole brain radiotherapy for the treatment of newly diagnosed multiple brain metastases. Cochrane Database Syst Rev 2012; 4: CD003869.

4. Andrews DW, Scott CB, Sperduto PW, et al. Whole brain radiation therapy with or without stereotactic radiosurgery boost for patients with one to three brain metastases: phase III results of the RTOG 9508 randomised trial. Lancet 2004; 363: 1665-72.

5. Kondziolka D, Patel A, Lunsford LD, Kassam A, Flickinger JC. Stereotactic radiosurgery plus whole brain radiotherapy versus radiotherapy alone for patients with multiple brain metastases. Int J Radiat Oncol Biol Phys 1999; 45: 427-34.

6. Sanghavi SN, Miranpuri SS, Chappell R, et al. Radiosurgery for patients with brain metastases: a multi-institutional analysis, stratified by the RTOG recursive partitioning analysis method. Int J Radiat Oncol Biol Phys 2001; 51: 426-34.

7. Bauman G, Yartsev S, Fisher B, Kron T, Laperriere N, Heydarian M, VanDyk J. Simultaneous infield boost with helical tomotherapy for patients with 1 to 3 brain metastases. Am J Clin Oncol 2007; 30: 38-44.

8. Lagerwaard FJ, van der Hoorn EA, Verbakel WF, Haasbeek CJ, Slotman BJ, Senan S. Whole-brain radiotherapy with simultaneous integrated boost to multiple brain metastases using volumetric 
modulated arc therapy. Int I Radiat Oncol Biol Phys 2009; 75: 253-9.

9. Edwards AA, Keggin E, Plowman PN. The developing role for intensity-modulated radiation therapy (IMRT) in the non-surgical treatment of brain metastases. Br J Radiol 2010; 83: 133-6.

10. Liang X, Ni L, Hu W, Chen W, Ying S, Gong Q, Liu Y. A planning study of simultaneous integrated boost with forward IMRT for multiple brain metastases. Med Dosim 2013; 38: 115-6.

11. van't Riet A, Mak AC, Moerland MA, Elders LH, van der Zee W. A conformation number to quantify the degree of conformality in brachytherapy and external beam irradiation: application to the prostate. Int J Radiat Oncol Biol Phys 1997; 37: 731-6.

12. Shaw E, Kline R, Gillin M, Souhami L, Hirschfeld A, Dinapoli R, Martin L. Radiation Therapy Oncology Group: radiosurgery quality assurance guidelines. Int J Radiat Oncol Biol Phys 1993; 27: 1231-9.

13. Gutiérrez AN, Westerly DC, Tomé WA, Jaradat HA, Mackie TR, Bentzen SM, Khuntia D, Mehta MP. Whole brain radiotherapy with hippocampal avoidance and simultaneously integrated brain metastases boost: a planning study. Int I Radiat Oncol Biol Phys 2007; 69: 589-97.

14. RTOG Acute Radiation Morbidity Scoring Criteria. http://www. rtog.org/ResearchAssociates/AdverseEventReporting/AcuteRadiationMorbidityScoringCriteria.aspx.

15. RTOG/EORTC Late Radiation Morbidity Scoring Schema. http:// www.rtog.org/ResearchAssociates/AdverseEventReporting/LateRadiationMorbidity ScoringSchema.aspx.

16. Nakamura JL, Verhey LJ, Smith V, et al. Dose conformity of gamma knife radiosurgery and risk factors for complications. Int I Radiat Oncol Biol Phys 2001; 51: 1313-9.

17. Peñagarícano JA, Yan Y, Shi C, Linskey ME, Ratanatharathorn V. Dosimetric comparison of helical tomotherapy and Gamma Knife stereotactic radiosurgery for single brain metastasis. Radiat Oncol 2006; $1: 26$.

18. Tomita N, Kodaira T, Tachibana H, Nakamura T, Nakahara R, Inokuchi H, Shibamoto Y. Helical tomotherapy for brain metastases: dosimetric evaluation of treatment plans and early clinical results. Technol Cancer Res Treat 2008; 7: 417-24.

19. Rodrigues G, Yartsev S, Yaremko B, et al. Phase I trial of simultaneous in-field boost with helical tomotherapy for patients with one to three brain metastases. Int I Radiat Oncol Biol Phys 2011; 80: 1128-33.

\section{Address for correspondence}

\section{Xiaodong Liang}

Radiation Oncology Department

Hangzhou Cancer Hospital

Nanguan Avenue 34

310002 Hangzhou, PR China

e-mail: Ixdctopone@sina.com

Submitted: 5.03 .2013

Accepted: $\quad 30.10 .2013$ 